\title{
IMAGING THE DISK AND JET OF THE CLASSICAL T TAURI STAR AA TAU
}

\author{
Andrew W. Cox ${ }^{1}$, Carol A. Grady ${ }^{2,3}$, Heidi B. Hammel ${ }^{4,5}$, Jeremy Hornbeck ${ }^{6}$, Ray W. Russell ${ }^{7,11}$, \\ Michael L. SitKo ${ }^{8,9,11}$, AND Bruce E. Woodgate ${ }^{10}$ \\ ${ }^{1}$ Astronomy Department, Villanova University, Villanova, PA 19085, USA; acox02@ villanova.edu \\ ${ }^{2}$ Eureka Scientific, 2452 Delmer St., Suite 100, Oakland, CA 96402, USA \\ ${ }^{3}$ ExoPlanets and Stellar Astrophysics Laboratory, Code 667, Goddard Space Flight Center, Greenbelt, MD 20771, USA \\ ${ }^{4}$ AURA, 1212 New York Ave. NW, Suite 450, Washington, DC 20005, USA \\ ${ }^{5}$ Space Science Institute, Boulder, CO 80303, USA \\ ${ }^{6}$ Department of Physics and Astronomy, University of Louisville, Louisville, KY 40292, USA \\ ${ }^{7}$ The Aerospace Corporation, Los Angeles, CA 90009, USA \\ ${ }^{8}$ Department of Physics, University of Cincinnati, Cincinnati, OH 45221, USA \\ ${ }^{9}$ Space Science Institute, 4750 Walnut St., Suite 205, Boulder, CO 80301, USA \\ ${ }^{10}$ ExoPlanets and Stellar Astrophysics Laboratory, Code 667, NASA's Goddard Space Flight Center, Greenbelt, MD 20771, USA \\ Received 2012 September 14; accepted 2012 November 7; published 2012 December 13
}

\begin{abstract}
Previous studies of the classical T Tauri star AA Tau have interpreted the UX-Orionis-like photo-polarimetric variability as being due to a warp in the inner disk caused by an inclined stellar magnetic dipole field. We test that these effects are macroscopically observable in the inclination and alignment of the disk. We use Hubble Space Telescope (HST)/STIS coronagraphic imagery to measure the $V$ magnitude of the star for both STIS coronagraphic observations, compare these data with optical photometry in the literature, and find that, unlike other classical $\mathrm{T}$ Tauri stars observed in the same HST program, the disk is most robustly detected in scattered light at stellar optical minimum light. We measure the outer disk radius, 1"'15 \pm 0 ' 10 , major-axis position angle, and disk inclination and find that the inner disk, as reported in the literature, is both misinclined and misaligned with respect to the outer disk. AA Tau drives a faint jet, detected in both STIS observations and in follow-on Goddard Fabry-Perot imagery, which is also misaligned with respect to the projection of the outer disk minor axis and is poorly collimated near the star, but which can be traced $21^{\prime \prime}$ from the star in data from 2005. The measured outer disk inclination, $71^{\circ} \pm$ $1^{\circ}$, is out of the range of inclinations suggested for stars with UX-Orionis-like variability when no grain growth has occurred in the disk. The faintness of the disk, small disk size, and detection of the star despite the high inclination all indicate that the dust disk must have experienced grain growth and settling toward the disk midplane, which we verify by comparing the observed disk with model imagery from the literature.
\end{abstract}

Key words: circumstellar matter - instrumentation: high angular resolution - planetary systems - polarization protoplanetary disks - stars: individual (AA Tau)

Online-only material: color figure

\section{INTRODUCTION}

T Tauri (TTau) stars were first discovered in 1942 and found to have irregular photometric variability (Joy 1945, 1949). Subsequent studies have found that the variability can be sorted into activity related to accretion from the circumstellar disk, stellar activity, or occultation of the star by material in the disk (Herbst et al. 1994; Grinin et al. 1991). Natta \& Whitney (2000) predicted that occultation should be a consequence of how we view the star+disk system, or the inclination from the disk being face-on to our line of sight. For disks dominated by tiny dust grains that remain well mixed with the gas, they predict that large-amplitude light variability should be found for $45^{\circ}<i<65^{\circ}-68^{\circ}$ and that stars should be occulted for $i \geqslant$ $68^{\circ}$. A-stars with such variability are known and named after the class prototype UX Ori (UXOR; Grinin et al. 1991), but they are not as common as predicted by Natta \& Whitney (2000). T Tauri stars with this kind of variability are even rarer, prompting suggestions that additional effects, such as warps in the inner disk, might be required to produce occultation events (Terquem \& Papaloizou 2000).

One such classical T Tauri star (cTTS) is AA Tauri (spectral type $=\mathrm{K} 7 \mathrm{~V}-\mathrm{M} 0 \mathrm{~V}, d=140 \mathrm{pc}, T_{\text {eff }}=4000 \mathrm{~K}$, typical $\mathrm{A}_{V} \sim$

\footnotetext{
${ }^{11}$ Guest Observer, Infrared Telescope Facility.
}

0.8; Donati et al. 2010; Bouvier et al. 1999). The star is well studied, with a rotational period of 8.2 days (Vrba et al. 1993), $V$-magnitudes ranging from 12.3 to 14.3 (Grankin et al. 2007), and a $3 \mathrm{mag}$ range at $B$ (Simbad). The linear polarization of AA Tau is $0.6 \%-1.3 \%$ (Ménard et al. 2003) and is anti-correlated with the optical light. Bouvier et al. (2003) used the rotation of the star and $v \sin i$ to derive an inclination of $70^{\circ} \pm 10^{\circ}$ for the star. With this inclination, and assuming that the disk is composed of interstellar medium (ISM) like dust that is well mixed with the gas (Natta \& Whitney 2000), the star should be routinely occulted. O'Sullivan et al. (2005) modeled the photopolarimetric variability, assuming periodic occultation of the star by a warp in the disk, and found $i=75^{\circ} \pm 2^{\circ}$ for the inner disk, again using small dust grain models, which should result in the star being occulted for their derived inclination. Andrews \& Williams (2007), using sub-millimeter data, need large grains (sand to pebbles) to fit their data. Other studies have focused on misalignment of the magnetic and rotation axes for the star as a source of the warp (Bouvier et al. 1999; Terquem \& Papaloizou 2000; Donati et al. 2010).

These various studies, particularly the modeling efforts, have made inconsistent assumptions about the AA Tau system (e.g., grain opacity model and inclination predicting that star and inner disk should be occulted while using photometric 
Table 1

HST Journal of Observations

\begin{tabular}{lllllll}
\hline \hline Target & \multicolumn{1}{c}{ Date/Time } & ObsID & $\begin{array}{c}\text { Exp. } \\
(\mathrm{s})\end{array}$ & Aperture & $\begin{array}{c}\text { PA } \\
\left({ }^{\circ}\right)\end{array}$ \\
\hline AA Tau & 2001 Nov 7 10:17:57 & O6BX29010 & 2268 & wedgeA1.0 & 237.456 & $12.57 \pm 0.10$ \\
& 2002 Sep 5 16:44:23 & 06BX28010 & 2268 & wedgeA1.0 & 207.456 & $13.84 \pm 0.25$ \\
CI Tau & 2001 Aug 9 20:22:17 & o6bx35010 & 2268 & wedgeA1.0 & 218.06 & $13.01 \pm 0.3:$ \\
& 2002 Nov 9 04:17:45 & o6bx34010 & 2268 & wedgeA1.0 & 188.06 & $12.60 \pm 0.3:$ \\
DL Tau & 2001 Sep 8 20:58:21 & o6bx22010 & 2142 & wedgeA1.0 & 203.06 & $12.60 \pm 0.31$ \\
& 2001 Sep 8 22:34:36 & o6bx23010 & 2146 & wedgeA1.0 & 233.06 & $12.68 \pm 0.28$ \\
DM Tau & 2001 Dec 24 07:54:57 & o6bx24010 & 2159 & wedgeA1.0 & 0.06 & $13.66 \pm 0.24$ \\
& 2002 Jan 23 14:09:32 & o6bx25010 & 2159 & wedgeA1.0 & 33.94 & $13.76 \pm 0.25$ \\
DN Tau & 2001 Dec 21 10:59:31 & o6bx31010 & 2268 & wedgeA1.0 & 47.06 & $12.25 \pm 0.29$ \\
& 2002 Dec 27 13:32:33 & o6bx30010 & 2268 & wedgeA1.0 & 17.06 & $12.19 \pm 0.29$ \\
LkCa 15 & 2001 Oct 1 19:29:12 & ob6x36010 & 2268 & wedgeA1.0 & 206.06 & $11.92 \pm 0.27$ \\
& 2001 Oct 30 03:35:40 & ob6x37010 & 2268 & wedgeA1.0 & 236.06 & $11.94 \pm 0.29$ \\
\hline
\end{tabular}

and polarimetric variability to derive the inclination). All of these studies have used rather indirect techniques to probe the geometry, inclination, and conditions in the disk of AA Tau, such as modeling of the IR spectral energy distribution (SED; Robitaille et al. 2007) and use of photometric and polarimetric data (Bouvier et al. 1999, 2003; Ménard et al. 2003). An alternate approach is to use high angular resolution and high-contrast imagery to directly image the disk and its environment. Such data can be used to explore to what extent the disk shows signs of warping, and whether it is consistent with a highly flared structure or one where significant grain growth and settling must have occurred. In this study we use archival Hubble Space Telescope (HST) imagery of AA Tau and narrowband FabryPerot imagery obtained with the Goddard Fabry-Perot (GFP) to explore the properties of the disk and environment of AA Tau.

\section{OBSERVATIONS AND DATA REDUCTION}

\subsection{HST STIS Coronagraphic Data}

AA Tau was imaged with the STIS coronagraph on HST on two dates separated by 10 months as part of program HST-GO9136. After acquiring the star using the F28x50LP filter, the star was placed at the wedgeA1.0 aperture, using the coronagraphic wedge oriented along the detector $y$-axis at a point where the wedge is $1^{\prime \prime} 0$ in diameter. Each observation spanned the full remaining orbital visibility window and was executed as three sub-integrations to facilitate cosmic-ray removal. Only the lower half of the STIS CCD was read out for these observations, resulting in a $50^{\prime \prime} \times 25^{\prime \prime}$ field of view. The journal of observations is in Table 1. Initial STIS data reduction is as described in Grady et al. (1999).

When the star is not occulted by its circumstellar disk, additional steps must be taken to remove the stellar scattered and diffracted light to reveal the disk. Historically, two approaches have been taken for removal of the remaining stellar light: (1) roll-differencing (Lowrance et al. 2005) or (2) subtraction of light from a star similar to the target, but lacking a disk. In roll-differencing, a simplified version of what is now termed "angular differential imaging," after registration, an image taken with $H S T$ at one spacecraft roll angle is subtracted from data taken at a different roll angle. If the stellar magnitudes differ, then the subtracted image must also be scaled. STIS does not fully apodize the $H S T$ diffraction spikes, so scale factors can be derived when the diffraction spikes are nulled or minimized in the net image. For subtraction of an observation of a different star, selection criteria for point-spread function (PSF) template
Table 2

PSF Template Data

\begin{tabular}{lllllrl}
\hline \hline Star & ObsID & $V$ & $B-V$ & Date & $\begin{array}{c}\text { Exp } \\
(\mathrm{s})\end{array}$ & Program \\
\hline HR 3802 & o6bx47010-70 & 5.964 & 1.518 & 2001 Oct 13 & 65.5 & 9136 \\
HR 2662 & o6bx46010-70 & 5.17 & 1.40 & 2001 Oct 12 & 37.6 & 9136 \\
HR 4176 & o6bx45010-70 & 5.746 & 1.333 & 2001 Oct 16 & 57.4 & 9136 \\
HR 957 & o6bx44010-70 & 6.254 & 1.201 & 2001 Sep 13 & 100.0 & 9136 \\
HD 85512 & o64w05010-30 & 7.651 & 1.179 & 2000 Nov 3 & 416.0 & 8624 \\
HD 154363 & o69x03010-50 & 7.709 & 1.172 & 2007 Jul 5 & 336.0 & 8442 \\
HR 2437 & o6bx42010-60 & 6.117 & 0.976 & 2001 Aug 12 & 86.4 & 9136 \\
\hline
\end{tabular}

data include a reasonable color match to the science target, no history of photometric variability for the template star, and no detected infrared excess (Grady et al. 2003). For our study we used PSF template star data taken as part of HST-GO-9136 and more recent STIS coronagraphic observations (Table 2). The subtraction process is similar to roll-differencing when the magnitude of the template observation differs from the science observation but typically results in some residual banding along the diffraction spikes. This is a consequence of color differences between the science target and the PSF template star and the wide bandpass of the unfiltered STIS CCD.

\subsection{Assembly of the IR SED}

In addition to extensive optical monitoring (Vrba et al. 1993; Herbst et al. 1994; Grankin et al. 2007), AA Tau has also been studied in the near-IR (NIR; Eisner et al. 2007), but not typically at the same time. We have used UBVRI photometry of Vrba et al. (1993) and JHKs photometry of Eisner et al. (2007) to establish the full amplitude of the photometric variability. The UBVRI observations from 1985 October to December were obtained with a variety of telescopes using both Johnson and Cousins-Kron RI filters, but all of the Johnson photometry was transformed to the Cousins-Kron system by the authors of the cited papers. The JHKs data were obtained in 2004 and 2005 October-November using the Peters Automated Infrared Imaging Telescope with Two Micron All Sky Survey (2MASS) instrument (Bloom et al. 2006). These data are supplemented by mid-IR data from IRAS (1980s), AKARI (Takita et al. 2010), and WISE (Wright 2009), as well as Spitzer Infrared Spectrograph (IRS) data, previously discussed in Furlan et al. (2006), and a Broadband Array Spectrograph System (BASS) spectrum from 2007. BASS spectra and their reduction are discussed in 
Table 3

AA Tau $V$ Magnitudes

\begin{tabular}{llcllc}
\hline \hline AA Tau & PSF Template & Scale Factor & $\Delta m$ & $V_{\text {PSF }}$ & $V$ \\
\hline 2001 & HD 154363 & $0.01005 \pm 0.0001$ & 4.995 & 7.73 & 12.72 \\
& HR 4196 & $0.0018 \pm 0.0001$ & 6.86 & 5.75 & 12.61 \\
& HR 2662 & $0.001155 \pm 0.001$ & 5.17 & 7.34 & 12.51 \\
& HR 3802 & $0.00215 \pm 0.001$ & 5.96 & 6.69 & 12.65 \\
& HR 957 & $0.003 \pm 0.0005$ & 6.25 & 6.31 & 12.56 \\
& HR 2437 & $0.003 \pm 0.0005$ & 6.12 & 6.31 & 12.43 \\
& & & & & $\langle 12.57 \pm 0.1\rangle$ \\
2002 & HR 4176 & $0.0006 \pm 0.0001$ & 5.75 & 8.055 & 13.8 \\
& HR 957 & $0.001 \pm 0.0005$ & 6.25 & 7.5 & 13.75 \\
& HR 2437 & $0.001 \pm 0.0005$ & 6.12 & 7.5 & 13.62 \\
& HD 88512 & $0.003 \pm 0.0005$ & 6.31 & 7.92 & 14.2 \\
& HR 2662 & $0.00034 \pm 0.0001$ & 5.75 & 8.67 & 14.42 \\
& & & & & $\langle 13.84 \pm 0.25\rangle$ \\
\hline
\end{tabular}

Kusakabe et al. (2012). Collectively, these data provide a wellsampled, composite IR SED from the optical through $40 \mu \mathrm{m}$.

\subsection{Goddard Fabry-Perot Data}

AA Tau was observed at the Apache Point Observatory $3.5 \mathrm{~m}$ with the GFP at Nasmyth focus on 2005 December 29 at [S II] $\lambda 6718.5$ using the Rutgers etalon, which transmits a velocity range of $134 \mathrm{~km} \mathrm{~s}^{-1}$ in a single observation (Wassell et al. 2006). The observations were carried out through variable cirrus. The [S II] observations were obtained as a partial velocity scan at $0 \mathrm{~km} \mathrm{~s}^{-1}$ (single exposure), $-100 \mathrm{~km} \mathrm{~s}^{-1}$ (two exposures), $-200 \mathrm{~km} \mathrm{~s}^{-1}$ (four exposures), and $-300 \mathrm{~km} \mathrm{~s}^{-1}$ (three exposures) with individual exposures of $900 \mathrm{~s}$. Images taken in this mode have default orientations of north up and east to the left. The velocity scan was taken with the star initially well centered under a coronagraphic wedge, but telescope pointing drifts caused the star to partially drive out of occultation toward the end of the observation set. These data were augmented by a short $R$-band target acquisition image, which allowed us to locate the center of light for AA Tau relative to field stars. Seeing was initially 2 ". 3 but further degraded during the velocity scan. Data reduction followed Wassell et al. (2006). Given the seeing and sky transparency conditions during the observations, and the AA Tau GFP data can map only the brightest portions of outflows, with sufficient velocity resolution to test whether an outflow component behaves like a jet, but cannot be used to address jet collimation or luminosity.

\section{RESULTS}

We use the HST STIS data to estimate the $V$ magnitude of AA Tau at the epoch of the coronagraphic observations and measure the orientation of the disk on the sky, the disk inclination, and the jet orientation. GFP data are used to measure the jet orientation at larger distances from AA Tau and constrain the jet radial velocity.

\subsection{Magnitudes for the STIS Observations}

The same process used to carry out PSF template observation subtraction can also be used to estimate the $V$ magnitude of AA Tau in both STIS observations. The effective wavelength of the unfiltered STIS CCD is $5875 \AA$, right in the middle of the $V$ band (Ely et al. 2011), so we add $\Delta m$ derived from the STIS data to the Simbad photometry for the template star to derive
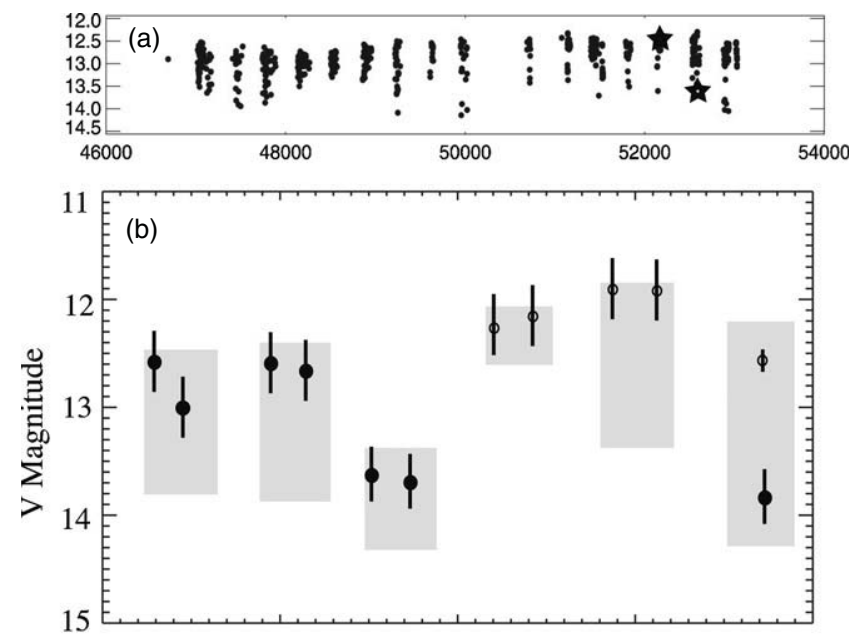

CI Tau DL Tau DM Tau DN Tau LkCa 15 AA Tau

Figure 1. Photometric Variability of AA Tau. (a) STIS $V$-magnitude equivalents (stars) compared with photometric data from Grankin et al. (2007). (b) STIS $V$-magnitude range of AA Tau compared with other T Tauri stars from HSTGO-9136. Magnitude ranges from Grankin et al. (2007) are shown as shaded boxes. Superposed on the literature range, we show the STIS equivalent $V$ magnitudes and their uncertainties. Filled symbols are shown where the disk has been firmly detected in scattered light (Grady et al. 2002a, 2002b), while open symbols indicate marginal or non-detections. Unlike the other T Tauri stars from this program, the firm STIS detection for AA Tau occurred near optical minimum light.

our estimate of $V$. Table 3 lists the scale factors, equivalent $\Delta m$, and the derived $V$-band magnitudes for AA Tau. The dominant source of error in this process is establishing whether we have the best null of the diffraction spikes feasible, given differences between AA Tau's spectrum and the spectra of the template stars. However, this source of error is reduced if we adopt the mean $V$ magnitude for measurements using an ensemble of PSF template stars. When averaged, the mean data have a dispersion of 0.1 for the 2001 data and 0.25 for the 2002 data. As a consistency check, we note that the magnitude estimate for the 2002 observation using the template stars is consistent with that derived from the magnitude estimate for the 2001 observation and the scale factor for the roll-differencing.

Grankin et al. (2007) published photometric data for AA Tau and several other T Tauri stars with similar STIS coronagraphy as part of a 20-year-long study of the photometric variability of T Tauri stars. For AA Tau, the data in Grankin et al. (2007) cover the epoch of the STIS observations, allowing us to compare our photometric estimates with the synoptic data. The 2001 data at $V \sim 12 \mathrm{~m} .6$ are 0 . 03 below the optical maximum light level for that year. The 2002 data are 0.04 below the minimum light for 2002, but within the historical range for AA Tau (Figure 1). AA Tau is unique among the T Tauri stars with STIS coronagraphy and photometry from Grankin et al. (2007) in having an HST observation near optical minimum. The remaining stars, which include disk detections (Grady et al. 2002a, 2002b, DL Tau, DM Tau) and non-detections (CI Tau, DN Tau, LkCa 15), were typically observed near their maximum light levels. Figure 1 shows where AA Tau and the other stars with photometry from Grankin et al. (2007) were in their light curves at the epoch of the STIS data.

\subsection{The Outer Disk of AA Tau}

AA Tau was observed with HST/WFPC2 on 1999 January 11. Inspection of the Hubble Legacy Archive data for both the F606W and F814W observations shows that the star dominates 

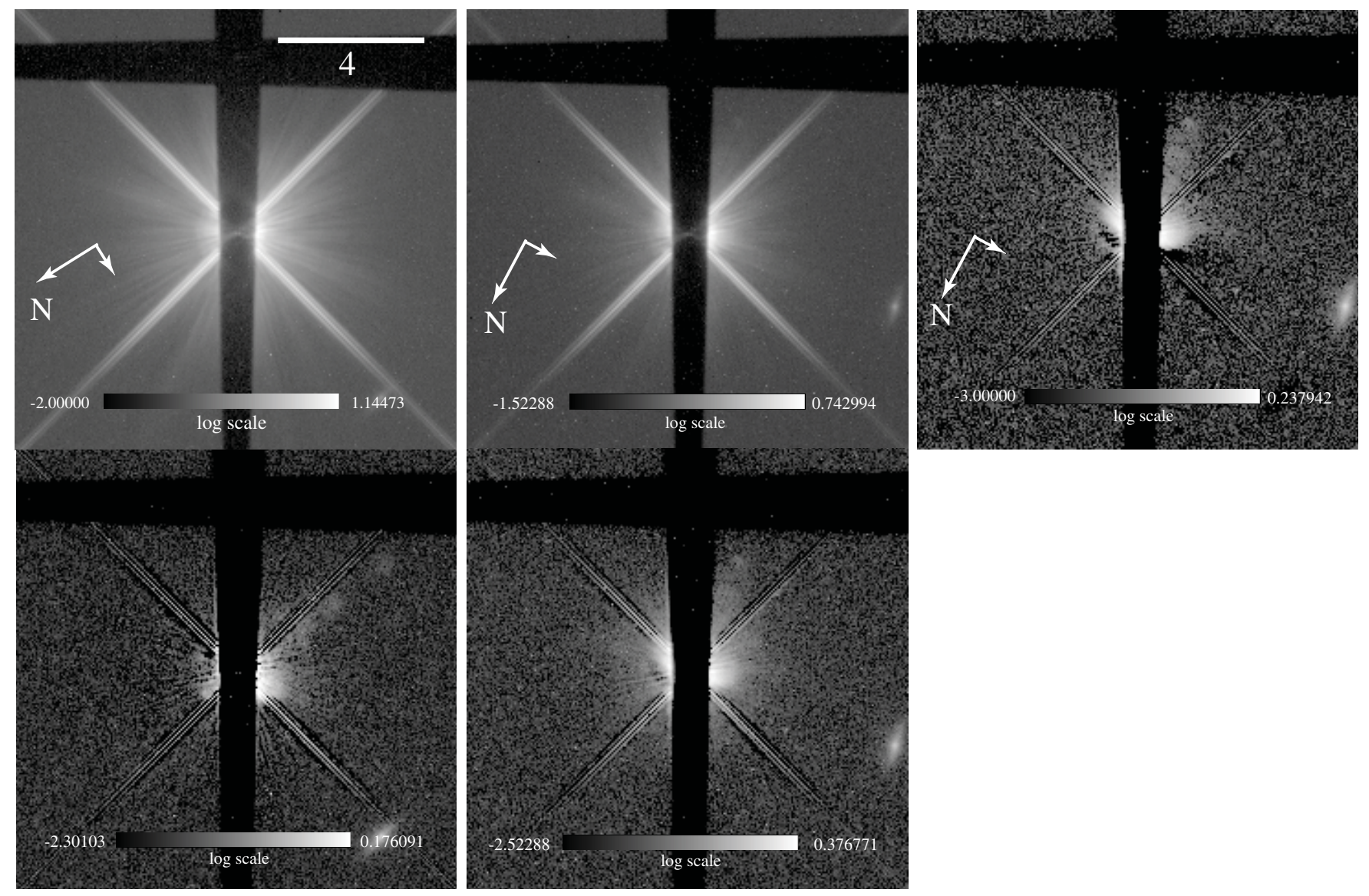

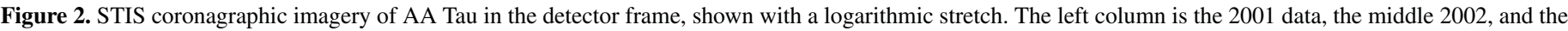
right column is 2002-2001, including the reduced, but not PSF-subtracted, imagery and the PSF-subtracted images. The field of view is $12^{\prime \prime}$ on a side.

the detected signal, in marked contrast to edge-on disk systems like HH 30 IRS $^{12}$ (Burrows et al. 1996; Watson \& Stapelfeldt 2007), where a dark lane flanked above and below by (relatively) bright regions of scattered light is observed. Other systems with similar nebulosity are all viewed at inclinations above $80^{\circ}$ from face-on (McCabe et al. 2011; Duchêne et al. 2010; Stapelfeldt et al. 2003; Wolf et al. 2003; Perrin et al. 2006; Hornbeck et al. 2012). In addition to the WFPC2 data, which clearly show that the star was detected, both STIS observations prior to PSF subtraction (Figure 2) show the conspicuous diffraction spikes expected for a stellar detection, suggesting that AA Tau must be viewed at $i<80^{\circ}$. Similarly, before subtracting the PSF template data, we do not see nebulosity in either STIS observation of AA Tau.

After PSF subtraction, nebulosity is seen near the star in the 2001 bright state (Figure 2), as well as a linear chain of blobs. In the 2002 faint state, a dark lane with flanking bright nebulosity similar to HH30 IRS is detected both with several of the PSF template observations and using roll-differencing. The PSF-subtracted faint state data also show the same chain of blobs seen in the bright state. We interpret the "hamburger-like" nebulosity as the circumstellar disk. When rotated to orient the PSF-subtracted imagery on the sky ( $\mathrm{N}$ up, E to left), the greatest disk elongation (major axis) is close to but not quite horizontal. A further rotation of $7^{\circ} \pm 2^{\circ}$ places the disk major axis closer to horizontal, indicating that the outer disk majoraxis position angle (PA) is $97^{\circ} \pm 2^{\circ}$. Adopting this value, we

12 For an image see http://hubblesite.org/newscenter/archive/releases/ 2000/32/image/c/ calculate that the projection of the minor axis should lie along $\mathrm{PA}=7^{\circ}$ or $187^{\circ} \pm 2^{\circ}$. In STIS imagery, where the stellar diffraction spikes are incompletely anodized, the star can be located under the coronagraphic wedge by extrapolating the location of the residual diffraction spikes into the wedge (e.g., $\mathrm{X}$ marks the spot) to typically \pm 1 pixel $(0.05$ or 7 AU at $d=$ $140 \mathrm{pc}$ ). Using this estimate of the stellar location and with the image rotated to place the disk horizontal, we estimated the disk outer radius from the farthest extent from the star where we could confidently trace the dark lane, or $r=1^{\prime \prime} .15 \pm 0$ "'.1 (156 \pm 14 AU assuming $d=140 \mathrm{pc}$ ) from the star.

If we assume that the disk is azimuthally symmetric, the dark lane should trace an ellipse that is the projection of a circle viewed at the inclination of the system. In Figure 3, we show the STIS roll-differenced imagery with the disk major-axis horizontal, and the brightest nebulosity up with a grid of such projected circles superposed. We can exclude inclinations below $68^{\circ}$ or above $75^{\circ}$ since these would place the dark lane tracing the disk midplane too far from the star (in projection) or too close to the star, respectively. The best-fit inclination is $71^{\circ} \pm 1^{\circ}$, which is in agreement with inclination estimates for the star (Bouvier et al. 2003), but with an order of magnitude smaller uncertainty. Our inclination measure differs from the inner disk measurement of $75^{\circ} \pm 2^{\circ}$ (O'Sullivan et al. 2005) by $2 \sigma$.

\subsection{Jet Imaging}

The chain of blobs seen in both PSF-subtracted STIS images is recovered in GFP imagery in [S II] obtained in late 2005 December, particularly at $-100 \pm 67 \mathrm{~km} \mathrm{~s}^{-1}$ and $-200 \pm$ $67 \mathrm{~km} \mathrm{~s}^{-1}$, but not in the rest of this velocity scan. The 


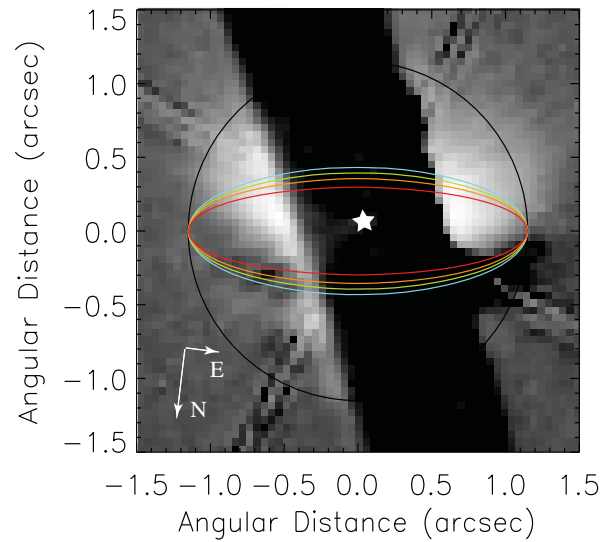

Figure 3. Constraining the inclination of the disk. The STIS roll-differenced image has been rotated to put the disk major axis on the horizontal. If the disk is azimuthally symmetric, the dark lane should trace the projection of a circle viewed at the system inclination. Projected circles are shown with the observed image, red $=75^{\circ}$ (traces top of dark lane), orange $=72^{\circ}$, yellow $=70^{\circ}$, blue $=$ $68^{\circ}$ inclination (traces bottom of dark lane). We derive $i=71^{\circ} \pm 1^{\circ}$.

(A color version of this figure is available in the online journal.)
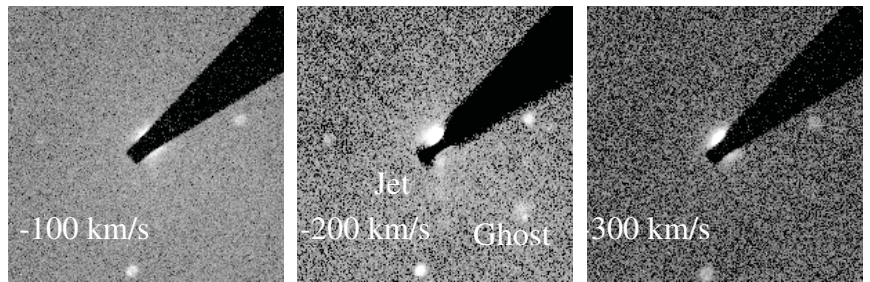

Figure 4. Goddard Fabry-Perot coronagraphic imagery of AA Tau shown in logarithmic stretch from 1 to 100 counts. The velocity scan in [S II] $\lambda 6717$ demonstrates that the knots are localized near $-200 \mathrm{~km} \mathrm{~s}^{-1}$, consistent with the expected behavior of a jet. The jet can be traced $\approx 21^{\prime \prime}(2920 \mathrm{AU})$ from AA Tau along $\mathrm{PA}=185^{\circ} \pm 4^{\circ}$. The field of view shown is $73^{\prime \prime}$ on a side and is oriented $\mathrm{N}$ up and $\mathrm{E}$ to left.

localization of the emission features in velocity demonstrates that the blobs are Herbig-Haro (HH) knots, while the linear geometry indicates detection of a jet (Figures 4 and 5). Stapelfeldt et al. (2003) note that a jet should be blueshifted on the side of the disk that is tipped toward us, and that this should correspond to the side of the disk with the brighter scattered light. This is exactly what we find. The jet can be traced almost 6" (840 AU) from the star in the STIS data. The PA of the jet was measured after rotating the STIS imagery to place north up and east to the left, tracing the center of light of the outflow in the 2001 data where the jet is better separated from the coronagraphic wedge. The HH knots in the STIS data are spatially resolved, and the 6" extent permits us to track the jet PA to \pm 1.5 along $\mathrm{PA}=183^{\circ}$. This PA differs from the jet PA predicted from the outer disk major-axis PA by $4^{\circ}(2.6 \sigma)$. Detection at larger distances in the STIS data is hampered by the placement of the coronagraphic structure relative to the jet, the fact that only the lower half of the STIS detector was read out, and sky background. Within $2^{\prime \prime}$ of the star, the jet opening half-angle is $17^{\circ}$ from the jet PA, and the jet is less well collimated than for other cTTS stars observed under the same $H S T$ program. In the $-200 \pm 67 \mathrm{~km} \mathrm{~s}^{-1}$ GFP imagery, $\mathrm{HH}$ knots can be traced $\approx 21^{\prime \prime}$ from the star along $\mathrm{PA}=185^{\circ} \pm 4^{\circ}$.

\section{DISCUSSION}

We have measured the inclination of the AA Tau outer disk to be $71^{\circ} \pm 1^{\circ}$, an order of magnitude more tightly constrained than the $70^{\circ} \pm 10^{\circ}$ measured for the star (Bouvier et al. 2003).
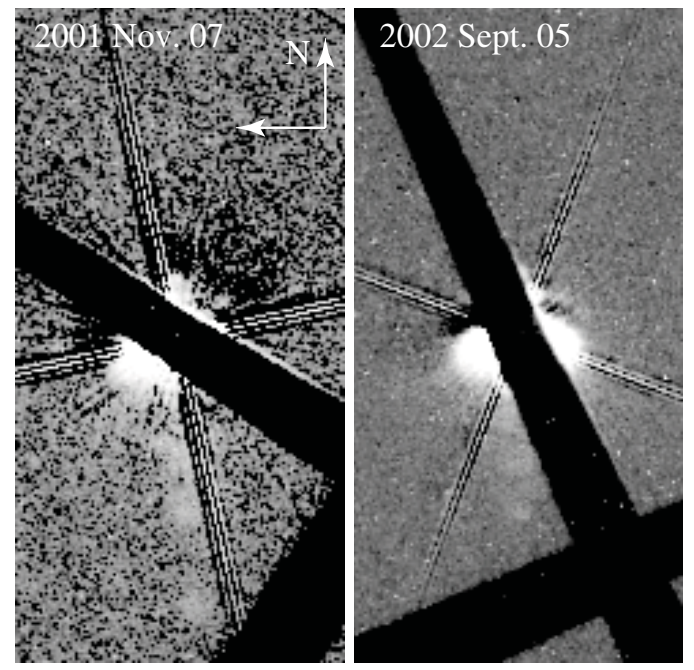

Figure 5. In addition to the disk, the STIS coronagraphic data at both epochs reveal a chain of knots extending along $\mathrm{PA}=183^{\circ} \pm 1.5$. The images are shown oriented on the sky ( $\mathrm{N}$ up, E to left) and show a region $6^{\prime \prime} \times 12^{\prime \prime}$ on a side. A possible counterjet is seen along $\mathrm{PA}=3^{\circ}$ within $2^{\prime \prime}$ of the star. The jet is less well collimated within $6^{\prime \prime}$ of the star than is typical of other single-star T Tauri stars. The rotation of the jet away from the polarimetrically predicted PA is highlighted by the vertical red line.

The outer disk major axis is along $\mathrm{PA}=97^{\circ} \pm 2^{\circ}$. Ménard et al. (2003) predicted from the polarization PA at minimum light that the disk major axis should be along $\mathrm{PA}=90^{\circ}$. Polarization measurements are dominated by dust closest to the star and thus reflect conditions in the inner disk. The majority of pre-mainsequence stars that drive jets have their jet PA orthogonal to the disk (Burrows et al. 1996; Stapelfeldt et al. 2003; Perrin et al. 2006; Grady et al. 2000; Wassell et al. 2006). For AA Tau, the jet PA near the star is offset by $4^{\circ}$ from where we would expect it to be based on the outer disk imagery. The jet is also less collimated within $6^{\prime \prime}$ of the star than typical of the jets detected in HST studies of single T Tauri stars.

\subsection{Disk Warp Is Macroscopically Detectable}

Robitaille et al. (2007) modeled AA Tau's SED and found that inclinations from $18^{\circ}$ to $80^{\circ}$ could fit the IR data. The SED alone constrains the disk inclination much less well than using the photometric rotation period, assumed size of the star, and $v \sin i$ (Bouvier et al. 2003). O'Sullivan et al. (2005) used the Bouvier inclination estimate and explored inclinations from $68^{\circ}$ to $75^{\circ}$. They also carried out Monte Carlo radiative transfer modeling of the outer disk and phase-resolved modeling of a warped inner disk. They derived an inclination of $75^{\circ} \pm 2^{\circ}$ for the inner disk. Our measurement of $i=71^{\circ} \pm 1^{\circ}$ demonstrates that the inner and outer disks are misinclined at the $2 \sigma$ level by $\approx 4^{\circ}$, while the outer disk PA and the PA inferred from the polarimetry suggest that the inner disk is also misaligned with the outer disk. The distortion of the inner disk appears to also be reflected in the misalignment of the jet with respect to the projection of the outer disk minor axis and the poor collimation of the jet near the star. Our data suggest that high-contrast imagery at wavelengths where jet emission can also be detected offers an efficient way to search for other systems with warps like AA Tau.

\subsection{Comparison with Sub-millimeter Data and Modeling}

Andrews \& Williams (2007) fit spatially resolved, submillimeter continuum interferometric visibilities and the SED 


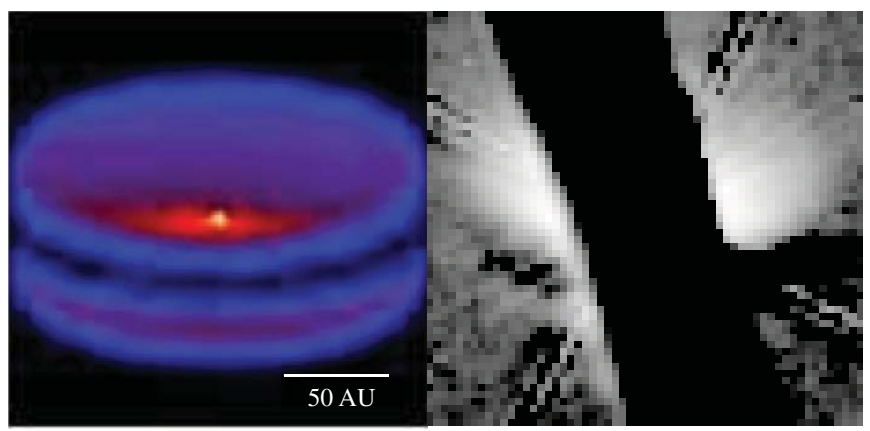

Figure 6. Comparison of the O'Sullivan et al. (2005) model for AA Tau viewed at $70^{\circ}$ with the STIS 2002 (minimum light) image rotated to align the horizontal axis with the system major axis. The model disk has a higher scale height at the outer radius than the observations, consistent with the actual disk having experienced grain growth and settling.

Table 4

Inclinations of UXORs with Imaged Disks

\begin{tabular}{lcl}
\hline \hline Star & $\begin{array}{c}\text { Inclination } \\
\left({ }^{\circ}\right)\end{array}$ & \multicolumn{1}{c}{ Reference } \\
\hline AA Tau & $71 \pm 1$ & This study \\
RY Tau & $65 \pm 4$ & McCleary et al. (2007) \\
HR 5999 & 58 & Preibisch et al. (2006) \\
PDS 144S & $73 \pm 7$ & Hornbeck et al. (2012) \\
\hline
\end{tabular}

of AA Tau, holding the inclination fixed at $75^{\circ}$. They derived $r_{\text {outer }}=400_{-75}^{+600} \mathrm{AU}$, which differs from our measurement for the scattered light disk of $r_{\text {outer }} \approx 160$ AU by $3 \sigma$. A $400 \mathrm{AU}$ disk viewed at $i \approx 70^{\circ}$ would place the dark lane marking the disk midplane farther from the star and can firmly be excluded by the HST data. The presence of a galaxy at 5.9 (826 AU) from AA Tau (Figure 2) not only provides a strict and extremely conservative upper limit to the optically thick portions of the AA Tau disk but suggests that the sub-millimeter data, both the continuum measurement and potentially the $\mathrm{CO}$ data (Öberg et al. 2010), may be confused with background objects, resulting in overestimates of the disk size. Higher angular resolution interferometric data, such as can be provided by ALMA, are required to assess the degree of confusion in the sub-millimeter.

Both Natta \& Whitney (2000) and O'Sullivan et al. (2005) model the outer disk with grain opacity laws dominated by small grains: such models predict that at $i=71^{\circ}$ the star should be occulted by its outer disk, in disagreement with the $H S T$ imagery. UXORs with optical or NIR high-contrast imagery or NIR to mid-IR interferometry are shown in Table 4 . These stars have inclinations $58^{\circ}<i<73^{\circ}$, including objects that meet expectations for disks dominated by small grains, but which are above the inclination limit where such model disks are expected to fully occult their stars. The shift in the inclination range where the UXOR phenomenon is observed to higher inclinations is also consistent with grain growth above interstellar values and should result in a disk that is geometrically flatter. Figure 6 shows a side-by-side comparison of the outer disk model from O'Sullivan et al. (2005) and the STIS imagery, with the disk major-axis horizontal in each image. The observed disk has scattered light below the dark midplane to only $50 \%$ of the height in the model disk. Thus, any small grain component of the disk must also have settled compared to Monte Carlo radiative transfer models using ISM-like grains. AA Tau adds to the body of data suggesting that the UXOR phenomenon can be observed in disks where grains must have grown and settled

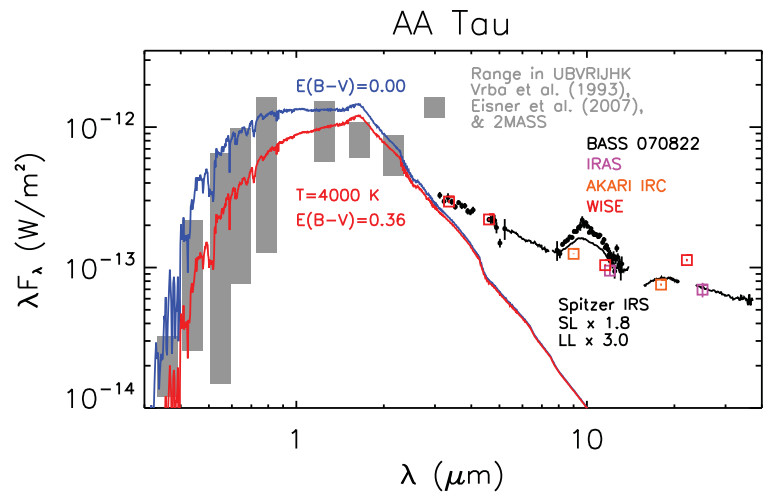

Figure 7. Optical and IR SED of AA Tau including data from Vrba et al. (1993), Eisner et al. (2007), 2MASS, IRAS, Akari IRC, WISE, Spitzer IRS, and BASS At $\lambda \leqslant 3 \mu \mathrm{m}$, AA Tau exhibits the large-amplitude photometric variability typical of UXORs (Grinin et al. 1991). The optical and NIR data can be fit with a $4000 \mathrm{~K}$ Kurucz model with no reddening at optical maximum light and an $R=3.1$ extinction curve at more typical optical light conditions. Gray boxes indicate the photometric range in the literature for AA Tau.

toward the disk midplane. We conclude that the disk of AA Tau has experienced grain growth and settling, to account for the lack of obscuration of the star by the outer disk and the reduced scale height of the disk compared to model imagery.

\subsection{Comparison with Other CTTS Disks Imaged with STIS}

Five other cTTS stars imaged in the same HST program as AA Tau also have photometry from Grankin et al. (2007). All five stars were imaged at or near optical maximum light (Figure 1), including both the disk detections and non-detections. The $V$-band photometric ranges for these stars are consistent with variability dominated by stellar activity or accretion variability. None of these other stars exhibit UXOR variability, which is consistent with their lower (more face-on) inclinations (Andrews \& Williams 2007). In contrast to these stars, AA Tau's disk was preferentially detected at optical minimum light, at a point where the warp provides additional suppression of the stellar light, but is sufficiently localized that the outer disk remains illuminated. A composite IR SED for AA Tau is shown in Figure 7, together with a reddened Kurucz (1991) model consistent with Donati et al. (2010). For this system, the star dominates the system light for $\lambda \leqslant 3 \mu \mathrm{m}$. We therefore expect that the pattern of preferential detection at minimum light would continue into the NIR wavelengths probed by HST/NICMOS and high-contrast imagers on $8-10 \mathrm{~m}$ telescopes such as HiCIAO (Hodapp et al. 2008) but would not continue into midIR imagery at $\lambda \geqslant 3 \mu \mathrm{m}$.

\section{SUMMARY}

We have established that the CTTS UXOR AA Tau was observed by HST/STIS near optical maximum light and near optical minimum. We have measured the system inclination, PAs for the disk major and minor axes, and disk outer radius. Our constraints on the system inclination are an order of magnitude tighter than Bouvier et al. (2003) and differ by $2 \sigma$, not in agreement with the inclination derived by O'Sullivan et al. (2005) for the inner disk. Similarly, the disk major axis is not in agreement with the value inferred from polarimetric data (Ménard et al. 2003). A jet is detected at both maximum and minimum light. The warp inferred to account for the UXOR photo-polarimetric variability of AA Tau manifests in changes 
in both inclination and alignment between the inner and outer disk, misalignment of jet with the outer disk semi-minor axis, and poor collimation of the jet within $2^{\prime \prime}$ of the star.

AA Tau is viewed at an inclination $i=71^{\circ} \pm 1^{\circ}$, which is inconsistent with its identification as a UXOR and history of photo-polarimetric variability (Grankin et al. 2007; Ménard et al. 2003) unless the dust disk has experienced grain growth and is settling toward the disk midplane. The imagery presented in this paper demonstrates that the disk has a smaller height than modeled by O'Sullivan et al. (2005), confirming that grain growth and settling must have occurred, to account for both the sub-millimeter data and the UXOR phenomenon at inclinations above those predicted by Natta \& Whitney (2000).

We wish to thank the anonymous referee for suggestions that have improved the paper and Edward Guinan for early readings of the manuscript. This work, in part, is based on observations made with the NASA/ESA Hubble Space Telescope, obtained at the Space Telescope Science Institute, which is operated by the Association of Universities for Research in Astronomy, Inc., under NASA contract NAS 5-26555. These observations are associated with program HST-GO9136. C.A.G. acknowledges support under HST-GO-9136 to Eureka Scientific. Additional support for the Goddard FabryPerot was provided by NASA RTOP 399131.02.02.02.32 and 399131.02.05.02.34 to Goddard Space Flight Center. Data used in this study were obtained at the Astrophysical Research Corporation (ARC) Apache Point Observatory $3.5 \mathrm{~m}$ telescope. APO Observing time was awarded as a grant of Directors Discretionary Time. NASA NNH06CC28C provided support for M.L.S., and NASA NNX09AC73G also supported C.A.G. and M.L.S.

\section{REFERENCES}

Andrews, S. M., \& Williams, J. P. 2007, ApJ, 659, 705

Bloom, J. S., Starr, D. L., Blake, C. H., Skrutskie, M. F., \& Falco, E. E. 2006, in ASP Conf. Ser. 351, Astronomical Data Analysis Software and Systems XV, ed. C. Gabriel et al. (San Francisco, CA: ASP), 751

Bouvier, J., Chelli, A., Allain, S., et al. 1999, A\&A, 349, 619
Bouvier, J., Grankin, K. N., Alencar, S. H. P., et al. 2003, A\&A, 409, 169 Burrows, C. J., Stapelfeldt, K. R., Watson, A.M, et al. 1996, ApJ, 473, 437 Donati, J.-F., Skelly, M. B., Bouvier, J., et al. 2010, MNRAS, 409, 1347

Duchêne, G., McCabe, C., Pinte, C., et al. 2010, ApJ, 712, 112

Eisner, J. A., Hillenbrand, L. A., White, R. J., et al. 2007, ApJ, 669, 1072

Ely, J., et al. 2011, STIS Instrument Handbook, Version 11.0 (Baltimore, MD: $\mathrm{STScI})$

Furlan, E., Hartmann, L., Calvet, N., et al. 2006, ApJS, 165, 586

Grady, C. A., Devine, D., Woodgate, B., et al. 2000, ApJ, 544, 895

Grady, C. A., Proffitt, C. R., Malumuth, E., et al. 2003, PASP, 115, 1036

Grady, C. A., Woodgate, B., Bruhweiler, F. C., et al. 1999, ApJL, 523, 151

Grady, C. A., Woodgate, B., Stapelfeldt, K., et al. 2002a, BAAS, 34, 764

Grady, C. A., Woodgate, B., Stapelfeldt, K., et al. 2002b, BAAS, 34, 1137

Grankin, K. N., Melnikov, S. Yu., Bouvier, J., et al. 2007, A\&A, 461, 183

Grinin, V., Kiselev, N. N., Chernova, G. P., Minikulov, N. Kh., \& Voshchinnikov, N. V. 1991, Ap\&SS, 186, 283

Herbst, W., Herbst, D. K., Grossman, E. J., \& Wienstein, D. 1994, AJ, 108,1906

Hodapp, K. W., Suzuki, R., Tamura, M., et al. 2008, Proc. SPIE, 7014, 701419

Hornbeck, J. B., Grady, C. A., Perrin, M. D., et al. 2012, ApJ, 744, 54

Joy, A. H. 1945, ApJ, 102, 168

Joy, A. H. 1949, ApJ, 110, 424

Kurucz, R. L. 1991, BAAS, 23, 1047

Kusakabe, N., Grady, C. A., Sitko, M. L., et al. 2012, ApJ, 753, 153

Lowrance, P. J., Bekclin, E. E., Schneider, G., et al. 2005, AJ, 130, 1845

McCabe, C. E., Duchêne, G., Pinte, C., et al. 2011, ApJ, 727, 90

McCleary, J., Stecklum, B., Grady, C., Woodgate, B., \& York, D. 2007, BAAS, 39, 849

Ménard, F., Bouvier, J., Dougados, C., et al. 2003, A\&A, 409, 163

Natta, A., \& Whitney, B. A. 2000, A\&A, 364, 633

Öberg, K. I., Qi, C., Fogel, J. K. J., et al. 2010, ApJ, 720, 480

O'Sullivan, M., Truss, M., Walker, C., et al. 2005, MNRAS, 358, 632

Perrin, M. D., Duchene, G., Kalas, P., et al. 2006, ApJ, 645, 1272

Preibisch, T., Kraus, S., Driebe, Th., van Boekel, R., \& Weigelt, G. 2006, A\&A, 458, 235

Robitaille, T., Whitney, B. A., Indebetouw, R., \& Wood, K. 2007, ApJS, 169,328

Stapelfeldt, K. R., Ménard, F., Watson, A. M., et al. 2003, ApJ, 589, 410

Takita, S., Kataza, H., Kitamura, Y., et al. 2010, A\&A, 519, A83

Terquem, C., \& Papaloizou, J. C. B. 2000, A\&A, 360, 1031

Vrba, F., Chugainov, P. F., Weaver, W. B., \& Stauffer, J. S. 1993, AJ, 106, 1608

Wassell, E. J., Grady, C. A., Woodgate, B., Kimble, R. A., \& Bruhweiler, F. C. 2006, ApJ, 650, 985

Watson, A., \& Stapelfeldt, K. 2007, AJ, 133, 845

Wolf, S., Padgett, D. L., \& Stapelfeldt, K. R. 2003, ApJ, 588, 373

Wright, E. L. 2009, in ASP Conf. Ser. 418, AKARI, a Light to Illuminate the Misty Universe, ed. T. Onaka, G. J. White, T. Nakagawa, \& I. Yamamura (San Francisco, CA: ASP), 359 